\title{
Preimplantation Genetic Testing for Aneuploidy Improves Live Birth Rates with In Vitro Produced Bovine Embryos: A Blind Retrospective Study
}

\author{
Giuseppe Silvestri ${ }^{1,+}+\mathbb{D}$, Carla Canedo-Ribeiro ${ }^{1,+}+\mathbb{D}$, María Serrano-Albal ${ }^{1,+}{ }^{,}$Remi Labrecque ${ }^{2}$, Patrick Blondin ${ }^{2}$, \\ Steven G. Larmer ${ }^{2}$, Gabriele Marras ${ }^{2}$, Desmond A.R. Tutt ${ }^{3}$, Alan H. Handyside ${ }^{1}$, Marta Farré ${ }^{1}$ (D), \\ Kevin D. Sinclair ${ }^{3}$ (I) and Darren K. Griffin ${ }^{1, *(1)}$ \\ 1 School of Biosciences, University of Kent, Canterbury CT2 7NH, UK; G.Silvestri@kent.ac.uk (G.S.); \\ cfcr2@kent.ac.uk (C.C.-R.); ms2104@kent.ac.uk (M.S.-A.); alan.handyside@outlook.com (A.H.H.); \\ m.farre-belmonte@kent.ac.uk (M.F.) \\ 2 L'Alliance Boviteq Inc., Saint-Hyacinthe, QC J2T 5H1, Canada; rlabrecque@boviteq.com (R.L.); \\ pblondin@boviteq.com (P.B.); slarmer@semex.com (S.G.L.); gmarras@semex.com (G.M.) \\ 3 School of Biosciences, University of Nottingham, Nottingham LE12 5RD, UK; \\ des.tutt@nottingham.ac.uk (D.A.R.T.); kevin.sinclair@nottingham.ac.uk (K.D.S.) \\ * Correspondence: d.k.griffin@kent.ac.uk \\ + These authors contributed equally to this work.
}

Citation: Silvestri, G.;

Canedo-Ribeiro, C.; Serrano-Albal, M.; Labrecque, R.; Blondin, P.; Larmer, S.G.; Marras, G.; Tutt, D.A.R.; Handyside, A.H.; Farré, M.; et al. Preimplantation Genetic Testing for Aneuploidy Improves Live Birth Rates with In Vitro Produced Bovine Embryos: A Blind Retrospective Study. Cells 2021, 10, 2284. https:// doi.org/10.3390/cells10092284

Academic Editor: Geert J.P.L. Kops

Received: 9 August 2021

Accepted: 30 August 2021

Published: 2 September 2021

Publisher's Note: MDPI stays neutral with regard to jurisdictional claims in published maps and institutional affiliations.

Copyright: (c) 2021 by the authors. Licensee MDPI, Basel, Switzerland. This article is an open access article distributed under the terms and conditions of the Creative Commons Attribution (CC BY) license (https:/ / creativecommons.org/licenses/by/ $4.0 /)$.

\begin{abstract}
Approximately one million in vitro produced (IVP) cattle embryos are transferred worldwide each year as a way to improve the rates of genetic gain. The most advanced programmes also apply genomic selection at the embryonic stage by SNP genotyping and the calculation of genomic estimated breeding values (GEBVs). However, a high proportion of cattle embryos fail to establish a pregnancy. Here, we demonstrate that further interrogation of the SNP data collected for GEBVs can effectively remove aneuploid embryos from the pool, improving live births per embryo transfer (ET). Using three preimplantation genetic testing for aneuploidy (PGT-A) approaches, we assessed 1713 cattle blastocysts in a blind, retrospective analysis. Our findings indicate aneuploid embryos have a $5.8 \%$ chance of establishing a pregnancy and a $5.0 \%$ chance of given rise to a live birth. This compares to $59.6 \%$ and $46.7 \%$ for euploid embryos $(p<0.0001)$. PGT-A improved overall pregnancy and live birth rates by $7.5 \%$ and $5.8 \%$, respectively $(p<0.0001)$. More detailed analyses revealed donor, chromosome, stage, grade, and sex-specific rates of error. Notably, we discovered a significantly higher incidence of aneuploidy in XY embryos and, as in humans, detected a preponderance of maternal meiosis I errors. Our data strongly support the use of PGT-A in cattle IVP programmes.
\end{abstract}

Keywords: cattle; breeding; single nucleotide polymorphism (SNP); mosaicism

\section{Introduction}

After humans (and possibly mice), the most studied mammalian species at the preimplantation stage of development (1-7 days post-fertilization) is cattle. In vitro production (IVP: the cattle equivalent of human IVF) techniques are used extensively for breeding purposes in order to shorten generation intervals and facilitate the bio-secure transportation of genetic resources [1]. The high demand for dairy and beef products has spurred the implementation of new approaches in IVP, combining the most recent advances in embryology with genomics and bioinformatics. Currently, around one million IVP cattle embryos are transferred annually, with a growing proportion screened for genomic estimated breeding values (GEBVs) before embryo transfer (ET) [2,3]. The idea of employing a genomic evaluation to estimate breeding values of individual animals is not new [4]. The in-depth characterisation of the bovine genome sequence has accelerated this trend, and has allowed the formulation of accurate models that describe the association between certain genetic markers (often SNPs: single nucleotide polymorphisms) and valuable traits. 
GEBVs have traditionally been determined soon after birth; however, modern screening at the embryonic stage has the advantage of shortening generation times further (to facilitate more rapid introduction of new genetics) and increasing selection intensity (by increasing the pool of screened individuals). In brief, the approach involves four stages: the biopsy of a portion of the trophectoderm, whole genome amplification (WGA), interrogation of a SNP array, and the subsequent bioinformatics analysis. The embryo transfer then proceeds as with any other IVP process. Currently in IVP, however, the proportion of live births per ET is still only around $50 \%$, depending on embryo quality and the production system employed (for a comprehensive review, see [5]). Moreover, there are no known GEBVs yet discovered that predict the chances of embryo implantation.

The presence of extra or missing chromosomes (principally aneuploidy) causes IVF failure in humans. Aneuploidy can originate during meiosis in either gamete (predominantly the oocyte) or in the embryo itself during the mitotic divisions of preimplantation development. The latter can give rise to mosaicism: a mixture of euploid and abnormal cells. Preimplantation genetic testing for aneuploidy (PGT-A), the practice of screening for chromosomal abnormalities prior to ET, has been the focus of much debate in the medical literature. Whilst the fact that there is a higher viability of euploid embryos seems to be well established, a recent randomised controlled trial gave mixed results, showing no overall benefit for PGT-A, except in older age groups [6]. This apparent paradox could be explained by a negative impact on embryo viability of the biopsy procedures (a pre-requisite for established PGT-A methods) or by misdiagnoses leading to embryo wastage [7]. Indeed, many cohort and retrospective studies have suggested that there is indeed a benefit of performing PGT-A [8].

Between 25 and $40 \%$ of cattle embryos produced by IVP carry a chromosomal abnormality [9-11], which (as in humans) can lead to early embryo development failure, pregnancy loss, or the birth of infertile animals. However, the use of PGT-A is not well established in animal breeding, and it has only recently been adapted to cattle screening, showing promising results [10]. Interrogating the SNP chip data to combine GEBV with PGT-A offers breeders the potential to achieve better embryo selection, and attain a higher chance of returning a pregnancy per embryo transferred.

Here, we made use of three methods of deriving aneuploidy information from SNP genotyped embryo biopsy material (discussed hereafter). The signal log $\mathrm{R}$ ratio (LRR) and B allele frequency (BAF) were calculated from the SNP intensity data to provide an accurate measure of the chromosome copy number [12]. Karyomapping [13] was employed to trace the parental origin of aneuploidy and the position of recombination events, as well as to diagnose those abnormalities that result in a normal karyotype, as is the case of the uniparental disomy (UPD). Finally, the meiotic or mitotic origin of trisomies was clarified by employing what we will term the Gabriel-Griffin algorithm [14].

Despite previous promising results $[10,11]$, the full benefit of PGT-A in cattle breeding has yet to be established. With this in mind, the purpose of this study was to test the hypothesis that a PGT-A strategy involving SNP chip interrogation significantly improves pregnancy and live birth rates for cattle IVP. The possible ramifications for human PGT-A are discussed.

\section{Methods}

\subsection{Study Design}

All data were acquired by L'Alliance Boviteq Inc. (Saint-Hyacinthe, QC, Canada) as part of their business operations. Ovarian stimulation, IVP, embryo biopsy, WGA, SNP genotyping, and embryo transfer were performed in a controlled commercial environment between 2016 and 2018. The resulting database compiled over this time consisted of 1737 transferred embryos and their 241 parents (168 dams and 73 sires), all of which were genotyped on a variety of different Illumina SNP chips as summarised in Table 1. Additionally, L'Alliance Boviteq Inc. kept records of parental age, and of embryo stage and grade, together with pregnancy outcomes following transfer. 
Table 1. SNP chips employed in this study. All chips were manufactured by Illumina (as part of their bovine range).

\begin{tabular}{cccc}
\hline SNP Chip Name & Number of SNPs & Embryos Tested (n) & Parents Tested (n) \\
\hline GGP Bovine HD 150k v01 & 138,892 & 379 & - \\
GGP Bovine HD 150k v03 & 139,376 & 1241 & - \\
GGP Bovine HD 150k v04 & 140,668 & 112 & - \\
GGP Bovine LD v04 & 30,105 & 5 & 241 \\
GGP Bovine SNP50 & 45,187 & - & \\
\hline "-" indicates no sample of this kind was tested on the corresponding platform.
\end{tabular}

The present study was conceived as a retrospective analysis of this database. The PGT-A investigation included $n=1713$ embryos derived from $n=73$ Holstein sires and $\mathrm{n}=168$ Holstein dams; 24 embryos were excluded from analysis as they lacked at least one full sibling or two half-siblings (which is a requirement for Karyomapping analysis). The PGT-A operators were blinded with regards to all information pertaining to parental age and history, as well as to any information related to embryo quality, stage, and outcomes following ET. This information was centrally retained by L'Alliance Boviteq Inc., and only disclosed after PGT-A analysis.

\subsection{Ovarian Stimulation and In Vitro Embryo Production}

Ovarian stimulation and IVP were carried out as described previously [15]. Briefly, $48 \mathrm{~h}$ following dominant follicle removal, dams underwent ovarian stimulation for 3 days, comprising six intra-muscular injections of FSH (Folltropin-V, Bioniche Animal Health, Belleville, ON, Canada) given at $12 \mathrm{~h}$ intervals, followed by a period of FSH withdrawal (coasting) typically lasting around $44 \mathrm{~h}$. Each injection contained 30 to $40 \mathrm{mg}$ of FSH (equivalent to 87.5 to $101.4 \mathrm{IU}$ ) at a manufacturer recommended concentration of $20 \mathrm{mg} / \mathrm{mL}$. Cumulus oocyte complexes (COCs) were recovered by ultrasound-guided follicular aspiration in warm HEPES-buffered Tyrode's medium with heparin $(10 \mathrm{IU} / \mathrm{mL})$. In vitro maturation (IVM) was carried out in HEPES-buffered media as previously described [10]. Maturing COCs were incubated for $24 \mathrm{~h}$ at $38.5^{\circ} \mathrm{C}$ in atmospheric $\mathrm{CO}_{2}$.

In vitro fertilisation was undertaken in $40 \mu \mathrm{L}$ droplets of modified Tyrode's lactate medium supplemented with $6 \mathrm{mg} / \mathrm{mL}$ BSA, $0.2 \mathrm{mM}$ pyruvate, $2 \mu \mathrm{g} / \mathrm{mL}$ heparin, $50 \mu \mathrm{g} / \mathrm{mL}$ gentamycin, and PHE [16]. The culture vessel was a Nunclon $35 \mathrm{~mm}$ cell culture dish (Nalgene Nunc, Rochester, NY, USA). Frozen spermatozoa were thawed and selected by discontinuous gradient centrifugation (BoviPure, Nidacon, Göthenborg, Sweden); the final sperm concentration in the fertilisation drops was $1 \times 10^{6}$ cells $/ \mathrm{mL}$ and the gametes were co-incubated at $38.5^{\circ} \mathrm{C}$ for 18 to $22 \mathrm{~h}$ under $5.5 \% \mathrm{CO}_{2}$. The resulting embryos were cultured in Nunclon dishes, using $50 \mu \mathrm{L}$ droplets of modified SOF medium supplemented with nonessential amino acids, $3 \mu \mathrm{M}$ EDTA, and $4 \mathrm{mg} / \mathrm{mL}$ BSA at $38.5^{\circ} \mathrm{C}$ under $6.5 \% \mathrm{CO}_{2}$ and $5 \% \mathrm{O}_{2}$.

\subsection{Embryo Biopsy, WGA, SNP Genotyping, and ET}

Embryos were evaluated as per the guidelines presented in [17]. Embryos of developmental stages 4 to 9 of either "Excellent" or "Good" morphology (grades 1 and 2) were washed and held in ViGRO flush solution (EGG Tech, Pullman, WA, USA), and approximatively 15 trophectoderm cells were biopsied using a micro-blade (Feather, Osaka, Japan). The biopsied cells were immediately subjected to whole genome amplification (WGA), which was carried out using a REPLI-g mini kit (Qiagen, Mississauga, ON, Canada) following the manufacturer's instructions. The resulting WGA products were then submitted for genotyping to a commercial service provider (GeneSeek (Neogen), Lincoln, NE, USA). The biopsied embryos were cryopreserved as previously described [10] before being transferred to suitably prepared recipient cows [18]. Clinical pregnancies were established at 60 days post ET by ultrasound. Hair samples from the parents were collected and sent directly to GeneSeek for a genotyping analysis. 


\subsection{Computing and PGT-A}

The SNP genotyping output files were archived by L'Alliance Boviteq Inc. The SNP database was then transferred to the University of Kent for a retrospective PGT-A analysis. Basic data manipulations on the database (cases extraction, file preparation for downstream analysis, filtering for shared SNPs) were performed using custom AWK and BASH scripts and resulted in the creation of output files for the PGT-A analysis. Each embryo included in the final study was analysed using a combination of three PGT-A algorithms, namely: signal intensity data LRR and BAF [12], Karyomapping [10,13], and Gabriel-Griffin plots [14]. Further details as relevant to each of these methods are presented below.

To detect the presence or absence of chromosome imbalances, LRR and BAF graphs were used, (discussed in [19]). The analysis pertaining to LRR and BAF was conducted in R [20] and figures were produced using the package karyoploteR [21]. Average R and Theta values for each $S N P$ combination $\left(R_{A A}, R_{A B}, R_{B B}\right.$, Theta $A A$, Theta $a_{A B}$, Theta $\left.a_{B B}\right)$ were calculated from raw signal intensity data $(X$ and $Y$ ) available from the SNP database itself; these values were then used to calculate the relevant data points on each graph (LRR and BAF) following the methodology described by Sun et al. [22]. For aneuploid cases, mosaicism diagnoses at the whole chromosome level were inferred as performed in Tutt et al. [11]. In segmental aneuploidy, only the affected part of the chromosome was used to calculate the mosaicism level for that aneuploidy.

To clarify the origin of each aneuploidy, two approaches were employed $[13,14]$ using in house VBA based algorithms. Output files containing the appropriate information for each sample (target embryo and its parents) were processed through the VBA macro BoVision (version 3.1, University of Kent, updated from [10]), and the VBA macro BoVisionGG (version 1.0, University of Kent).

\subsection{Limitations}

Whole chromosome and segmental errors were determined for all the samples in the study. However, chromosome Y errors could not reliably be investigated due to the limited number of SNPs available for this chromosome and the impossibility to implement Karyomapping's haploblock tracing. Similarly, paternal chromosome X errors could not be investigated by Karyomapping (sex chromosomes are present in single copy in the sire; therefore, haploblock tracing is prevented).

\subsection{Statistical Analysis}

The statistical analysis was completed on SPSS (version 26, IBM). Binomial data were compared by fitting a binomial or a multinomial generalised linear model with logit link functions. Multiple comparisons were performed using the Bonferroni correction. Continuous variables were investigated by ANOVA or a linear regression analysis as appropriate. The threshold for significance was set as $p=0.05$. Percentages were reported with their $95 \%$ confidence intervals (CI) for proportions. Pregnancy checks and live birth checks were performed for all the embryos transferred in this study $(n=1713)$. Birth weight checks were performed on $n=656$ out of a total $n=700$ live borne calves.

\subsection{Ethical Statement}

This study was performed entirely in silico by accessing information archived by L'Alliance Boviteq Inc. as a routine part of their business operations. As such, no live animals or embryos were employed.

\subsection{Data Availability Statement}

The SNP database analysed in this study is the intellectual property of L'Alliance Boviteq Inc. 


\section{Results}

\subsection{PGT-A Improves Pregnancy and Live Birth Outcomes}

The levels of aneuploidy were determined by LRR/BAF plots and Karyomapping which, when combined, discovered a chromosomal abnormality in $14.1 \%(n=241 / 1713)$ of the embryos. When ET outcomes were analysed, it was found that the embryos identified as euploid resulted in a pregnancy rate of $59.6 \%(\mathrm{n}=878 / 1472)$ and a live birth rate of $46.7 \%$ ( $\mathrm{n}=688 / 1472)$; in contrast, the embryos diagnosed as chromosomally abnormal resulted in a pregnancy rate of just $5.8 \%(n=14 / 241)$, and a live birth rate of $5.0 \%(n=12 / 241)$. In both cases, the difference was statistically significant $(p<0.0001)$. The associated odds ratios (OR) were 24.0 (95\% CI: 13.8-41.5) and 16.7 (95\% CI: 9.3-30.2) in favour of euploid embryos for pregnancy and live birth rates, respectively. The positive predictive value (PPV) for the type of PGT-A test we performed for live birth rates was therefore $98.3 \%$ (95\% CI: 97.1-99.0\%).

In the original database, the transfer of embryos without performing PGT-A led to a pregnancy rate of $52.1 \%(n=892 / 1713)$ and a live birth rate of $40.9 \%(n=700 / 1713)$, suggesting that the elective transfer of PGT-A selected euploid embryos would have improved pregnancy rates by $7.5 \%(p<0.0001, \mathrm{OR}=1.36)$ and live birth rates by $5.8 \%$ $(p=0.001, \mathrm{OR}=1.27)$. Importantly, from a breeder's perspective, the exclusive use of embryos diagnosed as euploid would have decreased the number of ETs required to obtain a live birth from 2.45 ETs/livebirth to 2.14 ETs/livebirth. A visual summary of these findings is given in Figure 1.

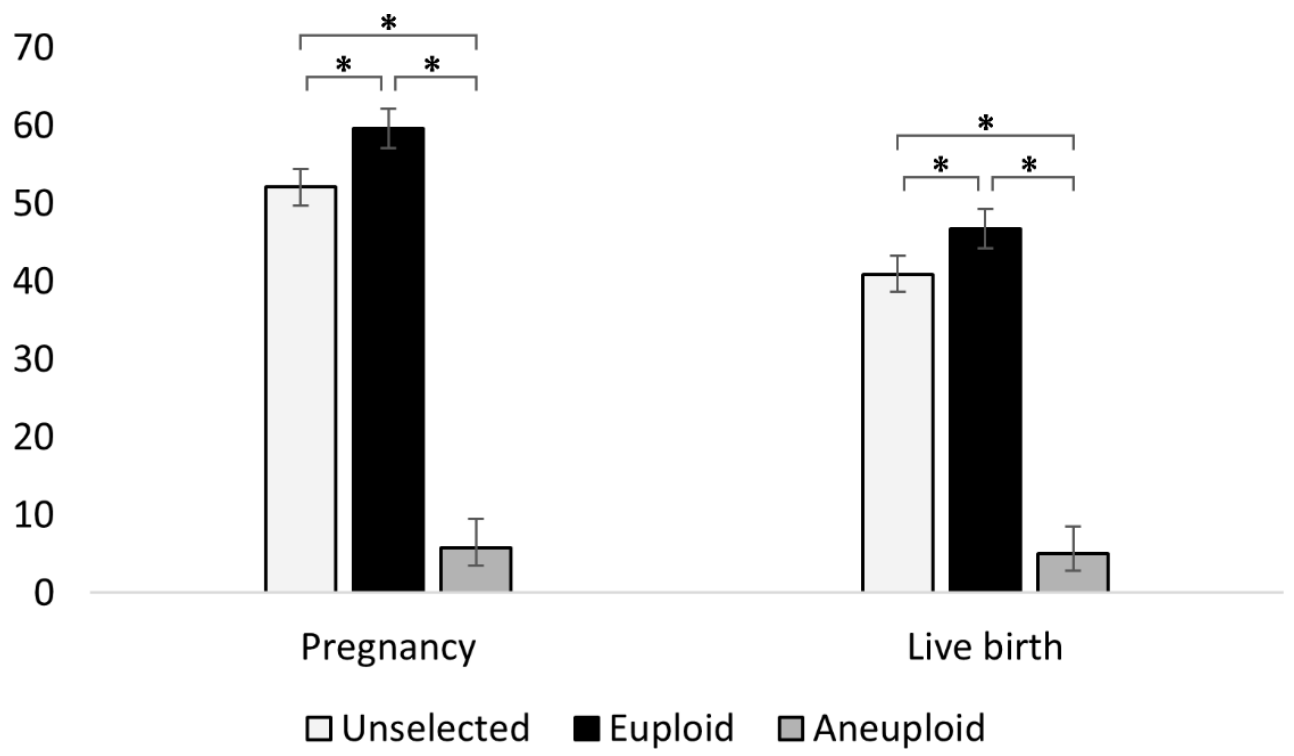

Figure 1. Effect of ploidy on pregnancy and live birth outcomes. Embryos diagnosed as euploid showed higher pregnancy and live birth rates as compared to embryos diagnosed as chromosomally abnormal or indeed to embryos not undergoing PGT-A selection. Sample population consisted of $\mathrm{n}=1713$ embryo transfers, which resulted in $\mathrm{n}=892$ pregnancies (unselected group); among these, retrospective PGT-A analysis discovered $n=878$ pregnancies from $n=1472$ euploid embryos and just $n=14$ pregnancies from $n=241$ aneuploid embryos. Similarly, there were a total of $n=700$ live births (unselected group), which were found to be derived from $n=688$ euploid embryos and $n=12$ aneuploid embryos. Data presented as percentage (\%) with $95 \%$ CI. * denotes statistically significant differences $(p<0.01)$.

The proportion of euploid embryos that successfully established a clinical pregnancy at D60 post transfer and then proceeded to yield a live birth was $78.4 \%(\mathrm{n}=688 / 878)$; conversely, among the few aneuploid embryos which also resulted in clinical pregnancies at D60, the live birth rate was $85.7 \%(n=12 / 14)$. The difference between these two proportions was not statistically significant $(p=0.51)$. Since no data were available on miscarriages 
within the first few weeks, we were not able to discriminate between early pregnancy loss (before D60) and embryo mortality. Nevertheless, with the limitations imposed by the small number of aneuploid embryos surviving in utero after D60, the data presented seem to suggest the presence of a bottleneck for aneuploid embryos positioned before D60 (be this either an early miscarriage or an embryo attachment failure), with the aneuploid embryos surviving this threshold behaving indistinguishably from the euploid embryos in terms of their ability to proceed to term.

\subsection{Chromosomal Abnormality Classes: Maternal Meiotic Errors Are by Far the Most Frequent}

The combination of PGT-A algorithms employed identified a range of abnormalities including whole chromosome and segmental errors and pinpointed their origin as being either meiotic or mitotic. Further to this, for all meiotic errors, we were able to detect whether the error originated in the maternal or paternal gamete. Additionally, for trisomies, we were also able to pinpoint the precise origin of the chromosomal error (meiosis I, meiosis II, mitosis) by Gabriel-Griffin plots. For monosomies, however, the same approach was unable to indicate whether the error originated in meiosis or during embryo development; instead, monosomies detected just in LRR/BAF plots but not by Karyomapping were classified as having originated de novo in the embryo (and therefore as being of mitotic origin). Overall, the most common abnormality class detected was monosomy, which appeared in $47.6 \%(n=139 / 292)$ of the errors. In this database, trisomies were slightly underrepresented as compared to monosomies, appearing in $38.7 \%(n=113 / 292)$ of the errors $(p=0.10)$. The remaining abnormality classes detected included segmental errors, triploidy/hypotriploidy, and uniparental disomy (UPD). These findings are presented in Table 2.

Table 2. Chromosomal abnormality class and frequency as identified by PGT-A. (A) Overview of all chromosomal errors detected, including a breakdown by error class and origin (maternal germline, paternal germline, embryonic). (B) Detailed overview of whole chromosome errors, providing a breakdown of error class (monosomy, trisomy, uniparental disomy (UPD)) and its origin; for trisomies, a further indication is provided to describe whether the error arose in meiosis I, in meiosis II, or during embryonic development (mitotic errors). In the case of UPDs, the origin is given as the parent passing two copies of its own chromosomes, or as the embryo in cases of a mosaic configuration.

\begin{tabular}{ccccc}
\hline (A) & & \multicolumn{3}{c}{ Origin } \\
\hline Aneuploidy class & Overall & Dam & Sire & Embryo \\
\hline Segmental errors & 21 & 2 & 14 & 5 \\
Triploidy and hypotriploidy & 16 & 6 & 10 & - \\
Whole chromosome & 255 & 212 & 16 & 27 \\
Total errors & 292 & 220 & 40 & 32 \\
\hline (B) & & & Origin & \\
\hline Whole chromosome & Overall & Dam & Sire & Embryo \\
\hline Trisomy & 113 & 90 & 1 & 22 \\
MI & 84 & 83 & 1 & - \\
MII & 7 & 7 & - & - \\
Mitotic & 22 & - & - & 22 \\
Monosomy & 139 & 120 & 15 & 4 \\
UPD & 3 & 2 & - & 1 \\
\hline u-" ind
\end{tabular}

"-" indicates no events of the corresponding origin were recorded.

Interestingly, the vast majority of errors for which a parental origin could be determined originated in the oocyte $(75.3 \%, n=220 / 292)$. More specifically, the trisomy analysis by the Gabriel-Griffin plots (for which a full breakdown is given in Supplementary Table S1) revealed that errors occurring during maternal meiosis I accounted for $73.5 \%$ of all trisomy events $(n=83 / 113)$, suggesting that a similar proportion of monosomies could have had the same origin. The only aneuploidy classes whose origins could be 
more prevalently ascribed to the paternal germline were triploidy/hypotriploidy (possibly resulting from polyspermy as well as or in place of meiotic errors), and segmental errors.

\subsection{Mosaicism Incidence, Pregnancy, and Live Birth Outcomes in Chromosomally Abnormal Embryos}

Among aneuploid embryos, at least $25.3 \%(n=61 / 241)$ carried one or more chromosomal abnormalities in a mosaic configuration. A detailed categorisation of these embryos, including their calculated mosaicism percentages is presented in Supplementary Table S2. Only $6.6 \%$ of ETs involving at least one mosaic error resulted in a live birth $(n=4 / 61)$; similarly, the live birth proportion among aneuploid non-mosaic cases was $4.5 \%(\mathrm{n}=8 / 179)$ and the difference between the two was not significant $(p=0.521)$. The average birth weight of calves born from mosaic and non-mosaic aneuploid cases (average of $42.7 \mathrm{~kg}$ and $38.9 \mathrm{~kg}$, respectively) was also not significantly different $(p=0.377)$, although the low sample size of these two groups may be masking any underlying effects. Additionally, the analysis is complicated by the fact that, on the field, parturition may be induced up to 10 days before the actual due date. With these limitations in mind, it is nevertheless interesting to note that the average birth weight did not differ between euploid and chromosomally abnormal embryos $(p=0.765)$. A detailed overview of the chromosomally abnormal embryos which still resulted in live births, is provided in Table 3. We were unfortunately unable to perform a follow-up study of these animals to confirm their ploidy status after birth.

Table 3. Live birth cases from aneuploid embryos. $\mathrm{Chr}=$ chromosome; UPD = uniparental disomy.

\begin{tabular}{|c|c|c|c|c|c|c|c|}
\hline Sample & Sex & $\begin{array}{c}\text { Embryo } \\
\text { Stage }\end{array}$ & $\begin{array}{c}\text { Embryo } \\
\text { Grade }\end{array}$ & Diagnosis & Chr & Mosaic & $\%$ Mosaicism \\
\hline 1536 & M & 6 & 1 & $\begin{array}{c}\text { Maternal } \\
\text { Monosomy }\end{array}$ & 4 & - & - \\
\hline 816 & $\mathrm{~F}$ & 5 & 2 & $\begin{array}{c}\text { Maternal } \\
\text { Monosomy }\end{array}$ & 22 & - & - \\
\hline 1173 & M & 7 & 1 & $\begin{array}{l}\text { Maternal } \\
\text { Trisomy }\end{array}$ & 4 & - & - \\
\hline 37 & M & 7 & 1 & $\begin{array}{l}\text { Maternal } \\
\text { Trisomy }\end{array}$ & 26 & - & - \\
\hline 1437 & M & 6 & 1 & $\begin{array}{l}\text { Maternal } \\
\text { Trisomy }\end{array}$ & 26 & - & - \\
\hline 1127 & M & 6 & 1 & $\begin{array}{l}\text { Maternal } \\
\text { Trisomy }\end{array}$ & 27 & - & - \\
\hline 1524 & M & 5 & 2 & $\begin{array}{l}\text { Mitotic } \\
\text { Trisomy }\end{array}$ & 6 & Yes & $76.0 \%$ \\
\hline 257 & M & 6 & 2 & $\begin{array}{l}\text { Segmental } \\
\text { Deletion }\end{array}$ & 17 & Yes & $47.3 \%$ \\
\hline 482 & $\mathrm{~F}$ & 6 & 2 & UPD & 6 & - & - \\
\hline 1021 & M & 8 & 1 & $\begin{array}{l}\text { Hypotriploidy } \\
\text { (polyspermy) }\end{array}$ & $\mathrm{n} / \mathrm{a}$ & Yes & 21.7 to $41.4 \%$ \\
\hline 353 & M & 6 & 2 & $\begin{array}{l}\text { Hypotriploidy } \\
\text { (polyspermy) }\end{array}$ & $\mathrm{n} / \mathrm{a}$ & - & - \\
\hline 1407 & M & 6 & 2 & $\begin{array}{l}\text { Hypotriploidy } \\
\text { (polyspermy) }\end{array}$ & $\mathrm{n} / \mathrm{a}$ & Yes & 21.0 to $47.0 \%$ \\
\hline
\end{tabular}

n/a = not applicable. "_" indicates the sample had a non-mosaic configuration, as such, no "\% mosaicism" is calculated.

\subsection{Parental Effects on Aneuploidy Incidence}

The average age of the donors $(n=168)$ was $10.3 \pm 0.7$ months. Sire age $(n=63)$ was recorded in groups (<10 months, 10-10.5 months, 10.5-11 months, 11-12 months, $>12$ months), with most males collected at either $10.5-11$ months $(n=9)$ or past 12 months of age $(n=12)$. The specific parent (dam or sire) employed in the IVP cycle seemed 
to influence the aneuploidy rate. Certain dams were more prone to produce aneuploid embryos $(p=0.0002)$; however, a sire specific effect was not discovered $(p=0.636)$. On the other hand, the age of the donor dam did not seem to have an effect on aneuploidy incidence $(p=0.678)$, a result which may not be surprising given the young average age of the dams employed. Curiously, we discovered a significant effect of sire age on aneuploidy incidence $(p=0.002)$, with a post-hoc investigation suggesting this result is due to a significant decrease in aneuploidy incidence associated with sperm collections performed when the sires were between 11 and 12 months of age. These observations are reported in Figure 2. Nonetheless, it should be noted that the relatively young age of all the parents employed prevents a comprehensive study of parental age effect on the incidence of aneuploidy as relevant to the wider breeding population.
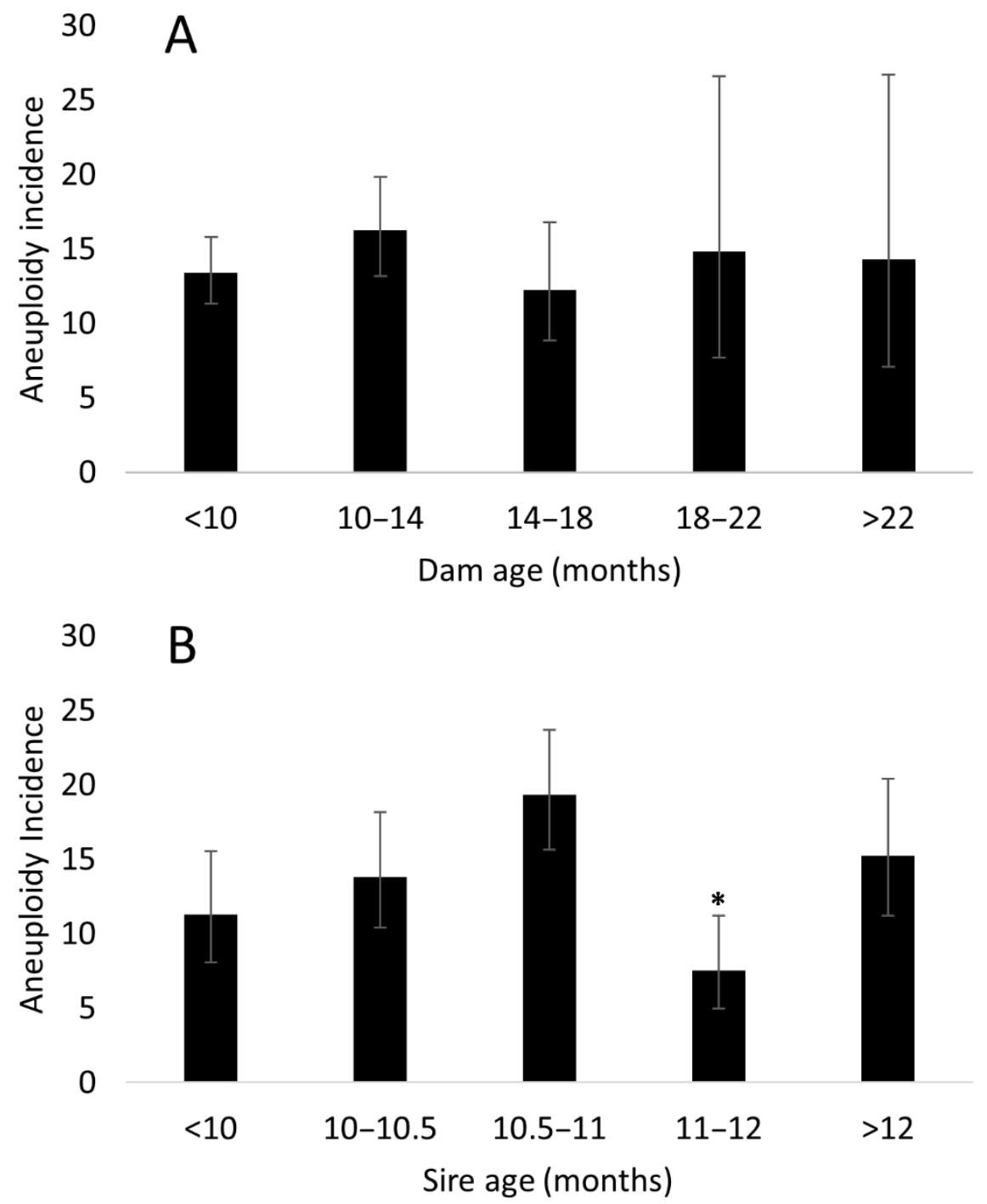

Figure 2. Embryo aneuploidy incidence by parent age. (A) Regression analysis showed no significant interaction between the age of the dam at oocyte collection and aneuploidy incidence in the resulting embryos $\left(R^{2}=0.0142, p>0.05\right)$. (B) Employing older sires did not negatively affect embryo ploidy; the star symbol $\left({ }^{*}\right)$ denotes a statistically significant $(p<0.01)$ aneuploidy incidence decrease for that group as compared to all others. Data presented as percentage (\%) with $95 \% \mathrm{CI}$.

\subsection{Relationship between Embryo Stage and Grade with Aneuploidy and Pregnancy/Live Birth Rates}

Interestingly, the overall aneuploidy incidence was significantly affected by the embryo's developmental stage at biopsy $(p<0.0001)$, with more advanced embryos displaying lower rates of chromosomal errors. This trend was also reflected in pregnancy and live 
birth outcomes, which tended to favour the embryos of later stages (Table 4). A more detailed analysis also revealed that the incidence of specific aneuploidy classes varied considerably within different embryo development stages, as reported in Figure 3A. In particular, the incidence of whole chromosome errors followed a trend where monosomies became significantly less prevalent at the more advanced developmental stages and trisomies and (hypo-)triploidies followed the opposite tendency. However, the incidence of segmental errors remained consistent across all the developmental stages investigated.

Table 4. Aneuploidy incidence, pregnancy, and live birth rates among embryos of different development stages. All three parameters were significantly affected by the embryo's development stage $(p<0.001)$. Only one stage 4 embryo was present in the database and it was therefore excluded from this analysis. Data are presented as mean with 95\% CI.

\begin{tabular}{ccccc}
\hline Embryo Stage & $\mathbf{n}$ & $\begin{array}{c}\text { Aneuploidy } \\
\text { Incidence (\%) }\end{array}$ & $\begin{array}{c}\text { Pregnancy Rate } \\
\text { D60 (\%) }\end{array}$ & Live Birth Rate (\%) \\
\hline 5 & 308 & $24.0(19.6-29.1)$ & $47.7(42.2-53.3)$ & $35.4(30.3-40.9)$ \\
\hline 6 & 786 & $15.0(12.7-17.7)$ & $50.5(47.0-54.0)$ & $41.0(37.6-44.4)$ \\
\hline 7 & 515 & $9.1(6.9-11.9)$ & $54.9(50.6-59.2)$ & $42.1(37.9-46.5)$ \\
\hline $8-9$ & 103 & $1.9(0.5-6.8)$ & $62.1(52.5-70.9)$ & $49.5(40.0-59.0)$ \\
\hline
\end{tabular}

Unsurprisingly, as was reported previously [11], we observed a link between embryo morphology and aneuploidy. The overall aneuploidy incidence in embryos classed as having grade 2 "good" morphology $(n=640)$ was $19.7 \%$, as opposed to just $10.7 \%$ in embryos classed as grade 1 "excellent" $(\mathrm{n}=1073)$, resulting in a significant difference $(p<0.0001)$. The different aneuploidy classes were also unequally represented between the two embryo grades, suggesting that embryo morphology is associated with not just the presence but also the type of aneuploidy. Interestingly, trisomies where overrepresented in the embryos of grade 1 where they affected $44.3 \%$ of the aneuploid embryos ( $n=51 / 115)$, as compared to $25.4 \%$ of the aneuploid grade 2 embryos $(p=0.002)$. Conversely, monosomies tended to affect the aneuploid grade 2 embryos more prominently with an incidence of $50 \%(n=63 / 126)$, as compared to $38.3 \%$ in the aneuploid grade 1 embryos $(n=44 / 115)$; however, this difference was not significant $(p=0.067)$. The other aneuploidy classes were equally distributed across the different embryo grades (as per Figure 3B).

Notably, following ET, grade 1 embryos achieved better pregnancy rates than grade 2 embryos (55.4\% versus $46.6 \%, p=0.0004)$. Unexpectedly, however, live births per embryo transferred were statistically similar between grade 1 and grade 2 embryos (41.8\% versus $39.4 \%, p=0.33$ ), suggesting that morphology alone may not be a faithful indicator of post transfer developmental potential.

\subsection{Male Embryos (XY) Achieve Higher Morphology Scores But Are Disproportionately Affected by Aneuploidy}

This database contained a significant excess of $\mathrm{XY}$ embryos as compared to $\mathrm{XX}$ embryos ( $\mathrm{n}=925$ males versus $\mathrm{n}=788$ female, $p=0.0009)$, as male embryos were more often required for transfer by the breeder. Interestingly, XY embryos tended to have better morphology, with $67.1 \%(n=621 / 925)$ of them being assigned to grade 1 , as compared to just $57.4 \%(n=452 / 799)$ of XX embryos $(p<0.0001)$. Additionally, we discovered that XY embryos had a higher incidence $(p=0.002)$ of chromosomal errors $(16.4 \%, \mathrm{n}=152 / 925)$ as compared to XX embryos $(11.3 \%, \mathrm{n}=89 / 788)$. On the other hand, the different aneuploidy classes were equally represented between sexes, as reported in Figure 4. This represents a novel observation in cattle, which is curiously at odds with what was just discussed in terms of embryo morphology and its relationship with sex. 

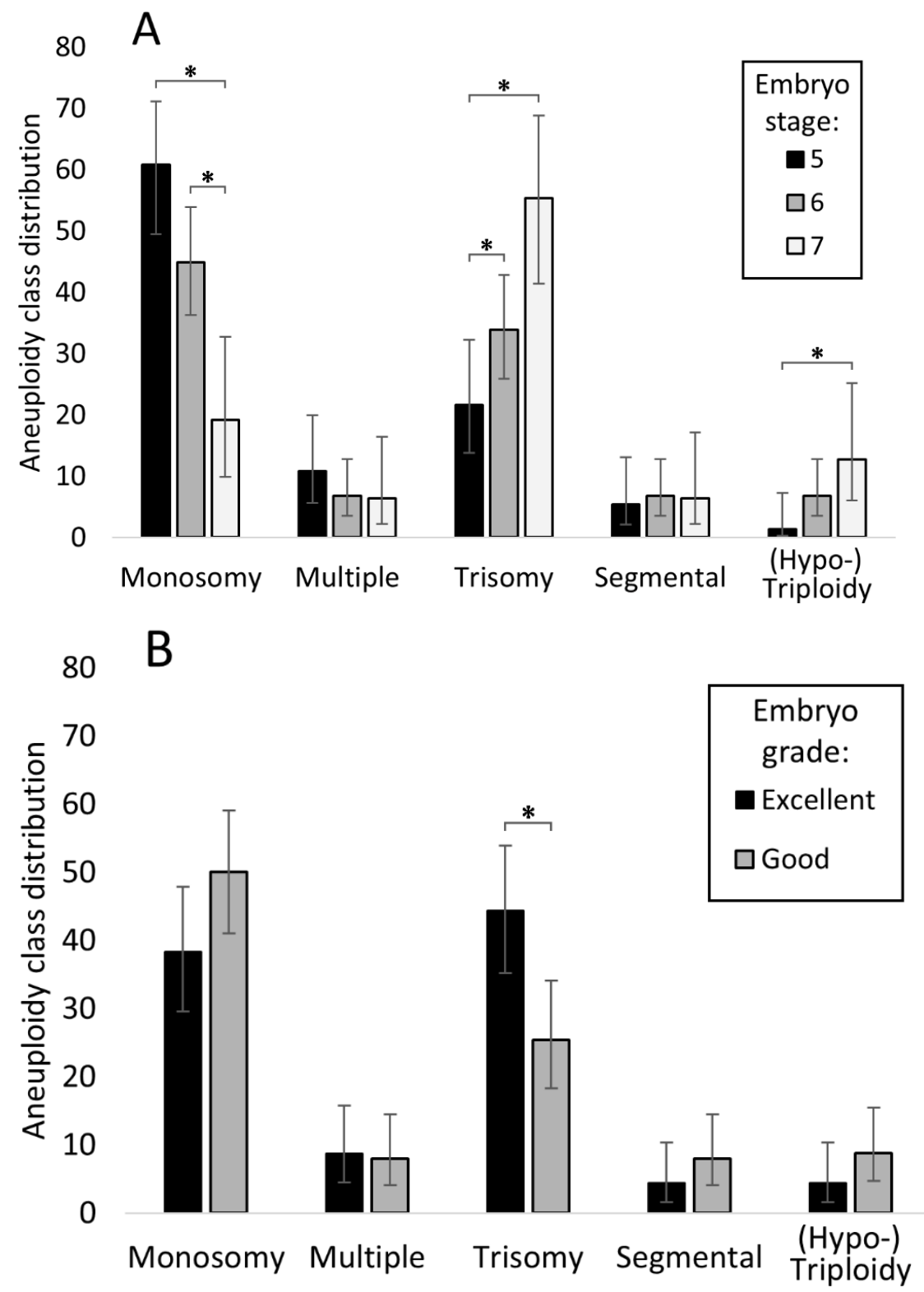

Figure 3. The distribution of aneuploidy types in embryos is affected by developmental stage and morphology grade. (A) The proportion of each aneuploidy class changes significantly across different developmental stages, with monosomy being the prevalent form at stage 5 and trisomy at stage 7 . Embryos of stages 4, 8, and 9 were omitted from this graph since samples size was less than three in each group. (B) Overall aneuploidy class distribution also differed between embryos of "Excellent" or "Good" morphology. Data presented as percentage (\%) with 95\% CI. * denotes statistically significant differences $(p<0.01)$.

\subsection{Incidence of Errors by Chromosome}

When triploidy/hypotriploidy events were excluded (a single event such as polyspermy which affects many chromosomes simultaneously), the chromosome specific incidence of aneuploidy seemed to vary considerably. Chromosomes 14 and 26 suffered the highest error frequencies ( $n=31 / 255$ and $n=18 / 255$, respectively), where chromosome 14 had the highest incidence of trisomies $(\mathrm{n}=21 / 31)$ and chromosome $26 \mathrm{had}$ a greater incidence of monosomies $(n=13 / 18)$. On the other hand, chromosome 7 did not show any type of error across the entire database. Of note, chromosomes 11, 13, 18, and 19 only showed trisomies, whilst chromosomes 21 and 22 showed only monosomies. A comprehensive summary of these results is given in Figure 5. 


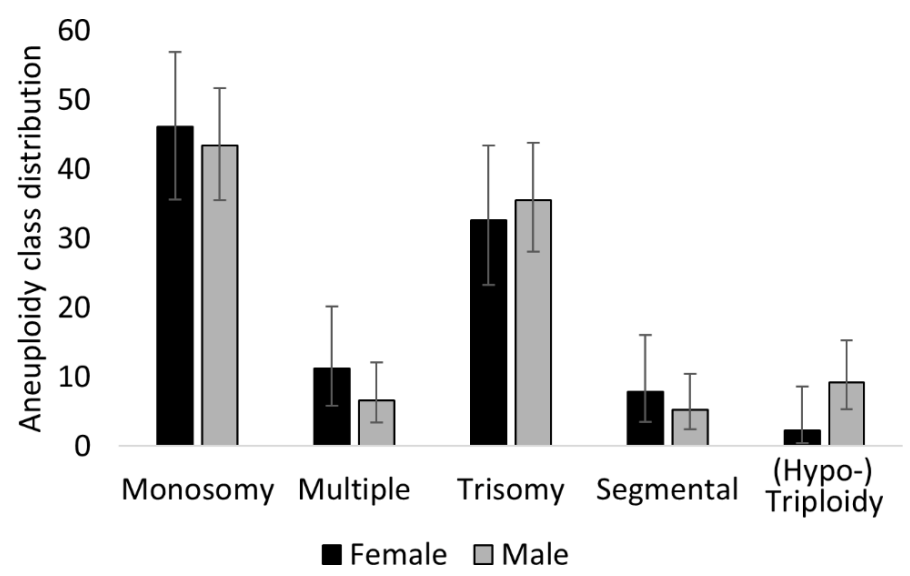

Figure 4. Relationship between embryo sex and aneuploidy. Although, overall, aneuploidy incidence was higher in $\mathrm{XY}$ embryos as compared to XX embryos, the different aneuploidy classes were equally distributed between them. Data presented as percentage (\%) with $95 \%$ CI.

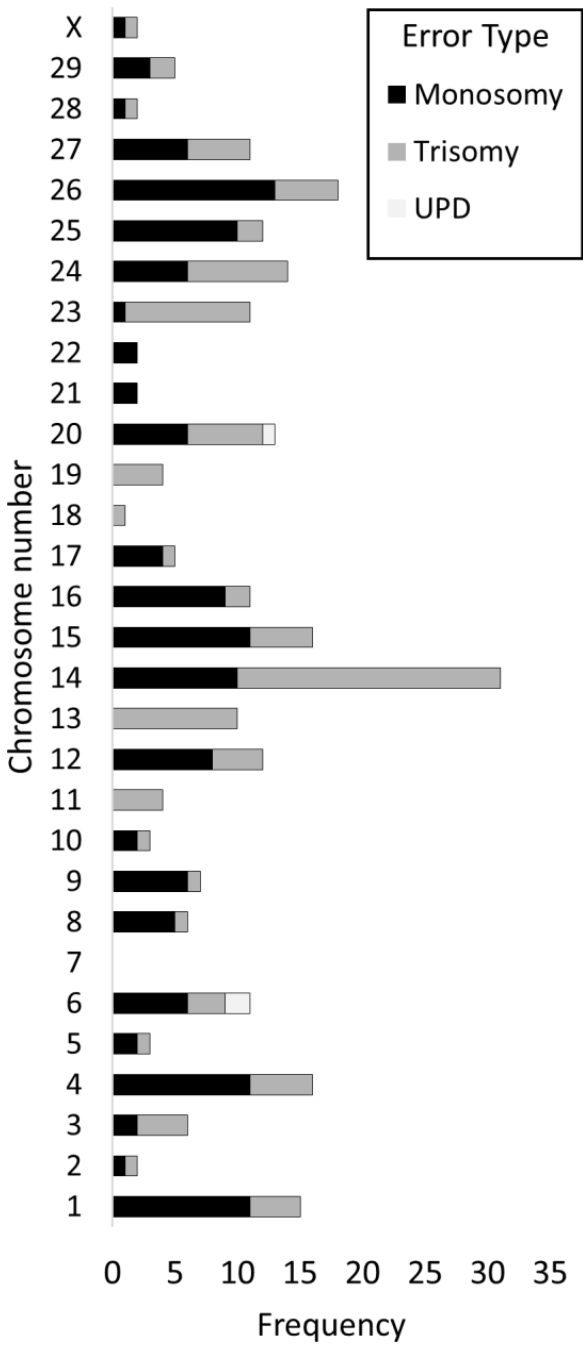

Figure 5. Whole chromosome error incidence by chromosome. Individual chromosomes were affected by aneuploidy at different rates, with specific chromosomes displaying a higher or lower incidence than expected. For example, monosomy and trisomy affected chromosome 14 at a much higher rate, whilst errors involving chromosome 7 were not detected in this database. $\mathrm{N}=255$ whole chromosome errors. 


\subsection{Chromosomal Loss in Hypotriploidy}

By analysing 15 cases of hypotriploidy (embryos with a less than complete triploid set of chromosomes), we observed a higher frequency of chromosome loss on chromosome $22(n=8 / 15)$ followed by chromosome $25(n=6 / 15)$, while chromosomes 6 and 17 did not show any chromosome loss. When chromosomes were arranged by size (measured in $\mathrm{Mb}$ ), a significant trend emerged suggesting that smaller chromosomes are more commonly affected by chromosome loss in hypotriploidy $(p=0.016)$, perhaps highlighting a mechanistic link between these two parameters. A visual representation of this analysis is proposed in Figure 6.

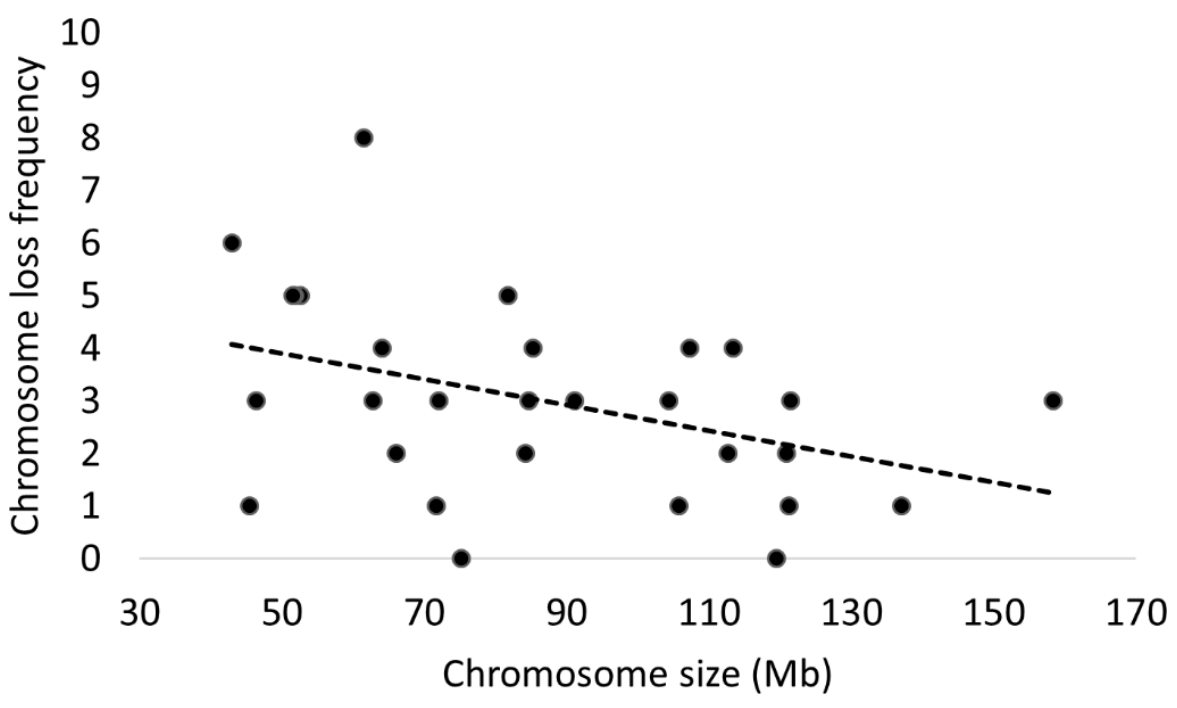

Figure 6. Frequency of chromosome loss in hypotriploidy. A significant trend was present, suggesting smaller chromosomes are more often lost in hypotriploidy $(p=0.016)$, even though the goodness of fit of this model was only modest $\left(\mathrm{R}^{2}=0.166\right)$.

\section{Discussion}

The results presented herein indicate that PGT-A is an effective selection strategy in cattle IVP, where embryos diagnosed as euploid are over 10 times more likely to implant than those diagnosed as aneuploid. Moreover, the exclusive use of embryos diagnosed as euploid significantly improves pregnancy and live births rates and would decrease the number of ETs required to obtain a live birth from 2.45 ETs/live birth to 2.14 ETs/live birth. This could potentially lead to significant financial savings as well as an improvement in efficiencies with environmental benefits (fewer dams needed on the ground to accept embryo transfers). The proposed strategy benefits from a high PPV (98\%) and seems particularly effective in the context of the modern cattle industry, which is increasingly reliant on IVP as a breeding technology [23] and has started implementing routine embryo biopsy and SNP genotyping for the imputation of GEBVs. As demonstrated here, in doing so breeders are already accumulating sufficient information for a comprehensive screen of chromosome abnormalities in their embryos, in addition to undertaking genomic selection.

\subsection{Relationship between Chromosomal Abnormality and Embryo Morphology}

The overall incidence of chromosome abnormalities detected in this study $(14.1 \%)$ is considerably lower than previously reported in cattle (30\% and higher, [9-11]). However, we suggest this lower figure is actually a substantial underestimation of the true overall incidence of chromosomal abnormalities following bovine IVP. This is due to the fact that this database only contains embryos pre-selected under strict morphological criteria by their breeder to maximise embryo biopsy survival, cryosurvival, and later live birth rates. Indeed, a link between embryo morphology and aneuploidy incidence has already been demonstrated [11,24], and this association is supported in our present work, especially 
for embryos biopsied at earlier developmental stages. Nonetheless, our findings strongly suggest an added benefit for PGT-A in addition to morphology screening in IVP, as we have shown that chromosomally abnormal embryos are still able to achieve the highest morphology scores. Furthermore, we present strong evidence that aneuploidy rates change significantly with the embryonic stage, with embryos still classified as blastocyst (stages 5 to 9) displaying widely different results. As such, we suggest that any aneuploidy incidence comparison between multiple studies should carefully consider the embryo developmental stage.

\subsection{Certain Chromosomes Are Preferentially Affected by Aneuploidy}

Whilst previous work by this group has already presented a breakdown of chromosome specific errors and aneuploidy classes affecting cattle embryos [10,11], the present study is more comprehensive in this respect thanks to the considerably larger sample size. We were able to highlight how certain cattle chromosomes are much more prone to aneuploidy (chromosome 14, in particular, followed by chromosome 26), while others (like chromosomes 2, 7, and 18) present very few or no errors. The distribution of errors between chromosomes challenges, at least in cattle, the observation that there is a reverse correlation between chromosome size and incidence of aneuploidy [25]. Instead, it suggests that chromosome specific properties might be worthy of consideration. However, a bias introduced by a different degree of lethality associated with aneuploidy affecting specific chromosomes cannot be discounted, since embryos were tested only at the blastocyst stage. We could imagine, for example, that a trisomy of chromosome 7 might almost always result in early developmental failure or severely compromised blastocyst morphology and thus was never detected in this study. This latter hypothesis is also supported by the presented evidence on chromosomal loss in hypotriploidy, where smaller chromosomes were indeed lost significantly more.

\subsection{Trisomic Embryos Survive Longer than Embryos Carrying a Monosomy}

Monosomies were the most frequent abnormality observed in this database followed by trisomies, a finding that compares well with recent trials in humans [6]. The fact that the number of trisomies is less than monosomies could be the consequence of a trisomy rescue producing euploid cells [26,27]. Nevertheless, we were also able to demonstrate that the relative frequency of certain aneuploidy types changes dramatically with the embryonic stage, with advanced embryos being more commonly affected by trisomies. This finding may not seem surprising when one considers that embryos tolerate trisomies better than monosomies. The extended culture of human embryos also confirmed that trisomies and duplications were more frequent than monosomies and deletions at later stages [28].

\subsection{Segmental and Mosaic Errors}

Segmental aneuploidies were also detected in this database. The transfer of embryos affected by these errors resulted in just one live birth (out of 21 transfers). A thorough investigation of the impact of this class of chromosomal abnormality remains challenging and may require a more substantial database. We detected mosaicism in at least a quarter of the embryos identified as chromosomally abnormal, but there was no difference between mosaic and non-mosaic embryos in terms of pregnancy and live birth. However, this result should be interpreted with caution given the very limited number of chromosomally abnormal embryos resulting in a live birth, and that SNP chip data (e.g., compared to next generation sequencing data) are limited in their ability to detect mosaicism. As such, the study of mosaicism in cattle IVP would certainly merit further exploration in the future.

\subsection{The Origin of Aneuploidy in Cattle IVP}

Interestingly, the vast majority of whole chromosome errors arose in the maternal germline and, in particular, the Gabriel-Griffin plot analysis suggested that $73.5 \%$ of trisomies occurred during meiosis I in the oocyte. Although reconstructing the meiotic 
origin of monosomy is not possible by Gabriel-Griffin plots, it could be speculated that a similar proportion of these errors would also have arisen in meiosis I. This analysis therefore confirmed that, in cattle oocytes, meiosis I appears to be more prone to chromosomal errors than meiosis II, in concordance with similar observations previously performed on human gametes $[19,29]$. Human oocytes are not routinely matured in vitro (contrarily to cattle); however, current evidence seems to suggest meiosis I is a critical step for aneuploidy in both organisms.

Previous work in the bovine model has identified significantly less aneuploidy in embryos derived from in vivo matured rather than IVM oocytes [30]. Within such a context, our results provide additional support for the findings of a recent study [11] which indicated that in vitro maturation of cattle oocytes (but not ovarian stimulation) is responsible for a significant proportion of the aneuploidy errors seen in IVP. The predominance of maternal meiotic errors in embryos from young donor females (where maternal age would not be a concern) implicates IVM as the step at which most chromosomal abnormalities arise, since meiosis is still in progress during oocyte culture.

\subsection{Differences between $X X$ and $X Y$ Embryos}

The incidence of aneuploidy differed significantly between $X X$ and $X Y$ embryos, with the male embryos being more severely affected. To the best of our knowledge, this is a novel finding and one which is in contrast to evidence collected in human IVF where a similar skew has not been observed [31,32]. Curiously, XY embryos tended to achieve better morphology grades; therefore, an IVP system relying solely on embryo morphology as a selection criterion may lead to an undesirable increase in the birth of male calves and may inadvertently contribute to the overall aneuploidy incidence in IVP (due to male embryos being more severely affected).

\subsection{The Wider Context}

PGT-A is a controversial subject in human reproductive medicine [8]. While around 70 retrospective analyses of single and multi-centre studies attest to its efficacy, randomised controlled trials returned mixed results, with only the older age group $(>35)$ showing consistently convincing beneficial effects [8]. The demonstrable physiological similarities with the human system make cattle a proxy for the study of human IVF. In particular, the bovine model we have investigated has the advantage that embryos were already committed to be biopsied and screened for a commercial necessity, removing a source of variability (the biopsy) with the breeder accepting any toll (however minor) such procedure might have on each embryo [7].

Nonetheless, it is important to note that in this work, as it is often routine in cattle, oocytes were in vitro matured before fertilization. However, IVM has found a much more limited use in human IVF. It has clearly been shown that embryos derived from IVM oocytes suffer from a higher incidence of chromosomal errors as compared to in vivo derived embryos $[11,30]$. As such, while a direct comparison between our data and PGT-A efficacy in clinical practice might be inappropriate, the bovine model can likely be useful for studying the impact of aneuploidy (of meiotic origin or otherwise) on pregnancy outcomes.

\section{Conclusions}

Overall, the data presented strongly support the use of PGT-A in cattle IVP where morphological grading of the embryos alone is not necessarily predictive of the embryo's potential to develop to term. Deselecting aneuploid cattle embryos in this way would lead to higher pregnancy and live birth rates per embryo transferred, resulting in a more cost efficient and environmentally sustainable system thanks to the reduced need for ET surrogates. The present findings add a new model to the body of evidence reporting a positive effect of PGT-A on ET outcomes in a system like cattle IVP, which is largely unaffected by issues of advanced maternal age but where in vitro maturation is routinely performed. Interestingly, all the embryos in this study were produced and biopsied at 
a single centre, thus reducing the methodological variation that may affect multi-centre studies and removing any possible bias introduced by the biopsy procedure itself between tested and untested embryos. The findings reported in this retrospective analysis should be followed up by a prospective study design where embryos are transferred after PGT-A based recommendations.

Supplementary Materials: The following are available online at https://www.mdpi.com/article/10 .3390/cells10092284/s1, Table S1: Trisomy origin.xlsx, Table S2: Aneuploidy and Mosaicism.xlsx.

Author Contributions: Conceptualization, G.S., D.A.R.T., K.D.S. and D.K.G.; methodology, G.S. and C.C.-R.; software, G.S., C.C.-R., S.G.L., G.M., A.H.H. and M.F.; formal analysis, G.S., C.C.-R., M.S.-A., K.D.S. and D.K.G.; investigation, G.S., C.C.-R., M.S.-A., R.L., P.B., S.G.L. and G.M.; resources, R.L., P.B., S.G.L., G.M., A.H.H. and M.F.; writing—original draft preparation, G.S., C.C.-R., M.S.-A., D.A.R.T., K.D.S. and D.K.G.; supervision, M.F., K.D.S. and D.K.G.; funding acquisition, K.D.S. and D.K.G. All authors have read and agreed to the published version of the manuscript.

Funding: This work was supported by the Biotechnology and Biological Sciences Research Council (BBSRC) LINK awards scheme (BB/R007985/1; BB/R00708X/1).

Institutional Review Board Statement: Ethical review and approval were waived for this study, due to the study being a retrospective analysis completed exclusively in silico.

Informed Consent Statement: Not applicable.

Data Availability Statement: Not applicable.

Acknowledgments: Specialist and high-performance computing clusters (ICARUS) were provided by Information Services at the University of Kent. The authors wish to thank Raffaele Silvestri for his assistance in developing parts of the VBA code required to run the analysis more efficiently.

Conflicts of Interest: The authors declare no conflict of interest.

\section{References}

1. Ferré, L.; Kjelland, M.; Strøbech, L.; Hyttel, P.; Mermillod, P.; Ross, P. Recent Advances in Bovine in Vitro Embryo Production: Reproductive Biotechnology History and Methods. Animal 2020, 14, 991-1004. [CrossRef]

2. Mullaart, E.; Wells, D. Embryo Biopsies for Genomic Selection. In Animal Biotechnology; Niemann, H., Wrenzycki, C., Eds.; Springer Nature: Cham, Switzerland, 2018; Volume 2, pp. 81-94.

3. Fujii, T.; Naito, A.; Hirayama, H.; Kashima, M.; Yoshino, H.; Hanamure, T.; Domon, Y.; Hayakawa, H.; Watanabe, T.; Moriyasu, S. Potential of Preimplantation Genomic Selection for Carcass Traits in Japanese Black Cattle. J. Reprod. Dev. 2019, 65, 251-258. [CrossRef]

4. Meuwissen, T.H.; Hayes, B.J.; Goddard, M.E. Prediction of Total Genetic Value using Genome-Wide Dense Marker Maps. Genetics 2001, 157, 1819-1829. [CrossRef]

5. Hansen, P.J. The Incompletely Fulfilled Promise of Embryo Transfer in cattle-Why aren't Pregnancy Rates Greater and what can we do about it? J. Anim. Sci. 2020, 98, skaa288. [CrossRef]

6. Munné, S.; Kaplan, B.; Frattarelli, J.L.; Child, T.; Nakhuda, G.; Shamma, F.N.; Silverberg, K.; Kalista, T.; Handyside, A.H.; Katz-Jaffe, M. Preimplantation Genetic Testing for Aneuploidy Versus Morphology as Selection Criteria for Single Frozen-Thawed Embryo Transfer in Good-Prognosis Patients: A Multicenter Randomized Clinical Trial. Fertil. Steril. 2019, 112, $1071-1079$. [CrossRef]

7. Pagliardini, L.; Viganò, P.; Alteri, A.; Corti, L.; Somigliana, E.; Papaleo, E. Shooting STAR: Reinterpreting the Data from the 'Single Embryo TrAnsfeR of Euploid Embryo'randomized Clinical Trial. Reprod. Biomed. Online 2020, 40, 475-478. [CrossRef]

8. Griffin, D.K.; Ogur, C. Chromosomal Analysis in IVF: Just how Useful is it? Reproduction 2018, 156, F29-F50. [CrossRef] [PubMed]

9. Hornak, M.; Kubicek, D.; Broz, P.; Hulinska, P.; Hanzalova, K.; Griffin, D.; Machatkova, M.; Rubes, J. Aneuploidy Detection and mtDNA Quantification in Bovine Embryos with Different Cleavage Onset using a Next-Generation Sequencing-Based Protocol. Cytogenet. Genome Res. 2016, 150, 60-67. [CrossRef]

10. Turner, K.J.; Silvestri, G.; Black, D.H.; Dobson, G.; Smith, C.; Handyside, A.H.; Sinclair, K.D.; Griffin, D.K. Karyomapping for Simultaneous Genomic Evaluation and Aneuploidy Screening of Preimplantation Bovine Embryos: The First Live-Born Calves. Theriogenology 2019, 125, 249-258. [CrossRef] [PubMed]

11. Tutt, D.; Silvestri, G.; Serrano-Albal, M.; Simmons, R.; Kwong, W.; Guven-Ates, G.; Canedo-Ribeiro, C.; Labrecque, R.; Blondin, P.; Handyside, A. Analysis of Bovine Blastocysts Indicates Ovarian Stimulation does Not Induce Chromosome Errors, nor Discordance between Inner-Cell Mass and Trophectoderm Lineages. Theriogenology 2021, 161, 108-119. [CrossRef] 
12. Attiyeh, E.F.; Diskin, S.J.; Attiyeh, M.A.; Mosse, Y.P.; Hou, C.; Jackson, E.M.; Kim, C.; Glessner, J.; Hakonarson, H.; Biegel, J.A.; et al. Genomic Copy Number Determination in Cancer Cells from Single Nucleotide Polymorphism Microarrays Based on Quantitative Genotyping Corrected for Aneuploidy. Genome Res. 2009, 19, 276-283. [CrossRef]

13. Handyside, A.H.; Harton, G.L.; Mariani, B.; Thornhill, A.R.; Affara, N.; Shaw, M.A.; Griffin, D.K. Karyomapping: A Universal Method for Genome Wide Analysis of Genetic Disease Based on Mapping Crossovers between Parental Haplotypes. J. Med. Genet. 2010, 47, 651-658. [CrossRef]

14. Gabriel, A.S.; Hassold, T.J.; Thornhill, A.R.; Affara, N.A.; Handyside, A.H.; Griffin, D.K. An Algorithm for Determining the Origin of Trisomy and the Positions of Chiasmata from SNP Genotype Data. Chromosome Res. 2011, 19, 155-163. [CrossRef] [PubMed]

15. Landry, D.A.; Bellefleur, A.; Labrecque, R.; Grand, F.; Vigneault, C.; Blondin, P.; Sirard, M. Effect of Cow Age on the in Vitro Developmental Competence of Oocytes obtained After FSH Stimulation and Coasting Treatments. Theriogenology 2016, 86, 1240-1246. [CrossRef]

16. Miller, G.; Gliedt, D.; Rakes, J.; Rorie, R. Addition of Penicillamine, Hypotaurine and Epinephrine (PHE) Or Bovine Oviductal Epithelial Cells (BOEC) Alone Or in Combination to Bovine in Vitro Fertilization Medium Increases the Subsequent Embryo Cleavage Rate. Theriogenology 1994, 41, 689-696. [CrossRef]

17. Bó, G.; Mapletoft, R. Evaluation and Classification of Bovine Embryos. Anim. Reprod. (AR) 2018, 10, $344-348$.

18. Blondin, P.; Farin, P.W.; Crosier, A.E.; Alexander, J.E.; Farin, C.E. In Vitro Production of Embryos Alters Levels of Insulin-Like Growth Factor-II Messenger Ribonucleic Acid in Bovine Fetuses 63 Days After Transfer. Biol. Reprod. 2000, 62, 384-389. [CrossRef]

19. Kubicek, D.; Hornak, M.; Horak, J.; Navratil, R.; Tauwinklova, G.; Rubes, J.; Vesela, K. Incidence and Origin of Meiotic Whole and Segmental Chromosomal Aneuploidies Detected by Karyomapping. Reprod. Biomed. Online 2019, 38, 330-339. [CrossRef]

20. R Core Team. R: A Language and Environment for Statistical Computing, 4.0.3; R Foundation for Statistical Computing: Vienna, Austria, 2020.

21. Gel, B.; Serra, E. KaryoploteR: An R/Bioconductor Package to Plot Customizable Genomes Displaying Arbitrary Data. Bioinformatics 2017, 33, 3088-3090. [CrossRef] [PubMed]

22. Sun, W.; Wright, F.A.; Tang, Z.; Nordgard, S.H.; Loo, P.V.; Yu, T.; Kristensen, V.N.; Perou, C.M. Integrated Study of Copy Number States and Genotype Calls using High-Density SNP Arrays. Nucleic Acids Res. 2009, 37, 5365-5377. [CrossRef]

23. Viana, J. 2017 Statistics of Embryo Production and Transfer in Domestic Farm Animals. Embryo Tech. News. 2018, $36,8-25$.

24. Alfarawati, S.; Fragouli, E.; Colls, P.; Stevens, J.; Gutiérrez-Mateo, C.; Schoolcraft, W.B.; Katz-Jaffe, M.G.; Wells, D. The Relationship between Blastocyst Morphology, Chromosomal Abnormality, and Embryo Gender. Fertil. Steril. 2011, 95, 520-524. [CrossRef]

25. Baryshnikova, A.; VanderSluis, B.; Costanzo, M.; Myers, C.L.; Cha, R.S.; Andrews, B.; Boone, C. Global Linkage Map Connects Meiotic Centromere Function to Chromosome Size in Budding Yeast. G3: Genes Genomes Genet. 2013, 3, 1741-1751. [CrossRef] [PubMed]

26. McCoy, R.C. Mosaicism in Preimplantation Human Embryos: When Chromosomal Abnormalities are the Norm. Trends Genet. 2017, 33, 448-463. [CrossRef] [PubMed]

27. Kahraman, S.; Cetinkaya, M.; Yuksel, B.; Yesil, M.; Pirkevi Cetinkaya, C. The Birth of a Baby with Mosaicism Resulting from a Known Mosaic Embryo Transfer: A Case Report. Hum. Reprod. 2020, 35, 727-733. [CrossRef]

28. Popovic, M.; Dhaenens, L.; Taelman, J.; Dheedene, A.; Bialecka, M.; De Sutter, P.; Chuva de Sousa Lopes, S.M.; Menten, B.; Heindryckx, B. Extended in Vitro Culture of Human Embryos Demonstrates the Complex Nature of Diagnosing Chromosomal Mosaicism from a Single Trophectoderm Biopsy. Hum. Reprod. 2019, 34, 758-769. [CrossRef] [PubMed]

29. Hassold, T.; Hunt, P. To Err (Meiotically) is Human: The Genesis of Human Aneuploidy. Nat. Rev. Genet. 2001, 2, 280-291. [CrossRef]

30. Tšuiko, O.; Catteeuw, M.; Zamani Esteki, M.; Destouni, A.; Bogado Pascottini, O.; Besenfelder, U.; Havlicek, V.; Smits, K.; Kurg, A.; Salumets, A.; et al. Genome stability of bovine in vivo-conceived cleavage-stage embryos is higher compared to in vitro-produced embryos. Hum. Reprod. 2017, 32, 2348-2357. [CrossRef] [PubMed]

31. Eaton, J.L.; Hacker, M.R.; Barrett, C.B.; Thornton, K.L.; Penzias, A.S. Influence of Embryo Sex on Development to the Blastocyst Stage and Euploidy. Fertil. Steril. 2011, 95, 936-939. [CrossRef]

32. Wang, A.; Kort, J.; Behr, B.; Westphal, L.M. Euploidy in Relation to Blastocyst Sex and Morphology. J. Assist. Reprod. Genet. 2018, 35, 1565-1572. [CrossRef] 